\title{
Bioelectronic Evolution in Developing Molecular and Cellular Biodevices
}

\author{
Masuo AIZAWA
}

\begin{abstract}
Department of Biological Information, Graduate School of Bioscience and Biotechnology, Tokyo Institute of Technology (Nagatsuta, Midori-ku, Yokohama 226-8501, Japan)
\end{abstract}

Received August 29, 2001 ; Accepted September 13, 2001

\begin{abstract}
Bioelectronics has been evoluted in development of biodevices through the biointerfacing technology, gene engineering, and cellular engineering. Several protein-based molecular biodevices have been made possible by the biointerfacing technology that enables proteins to electronically communicate with the electronic materials. Gene engineered proteins and cells led to develop innovative biodevices. Further bioelectronic evolution is in the process toward single-molecular biodevices and cellular biodevices.
\end{abstract}

Key Words : Biodevice, Biointerfacing, Cellular Biodevice

\section{Introduction}

Intensive efforts have long been targeted at realizing in vitro biological systems that are termed "biodevices" in either nano-scale or macro-scale with retaining the biological function of implemented bio-components. Biodevices are mostly designed to implement biological components such as protein molecules and living cells by imitating primarily the biological information systems that are capable of signal transduction and information processing. One of the ultimate goals is to design and to fabricate a neurobiodevice based on the brain. However, other biodevices with unique biological functions have been pursued, which include protein-based molecular biodevices such as biosensors. ${ }^{1-5}$ )

The original root of biodevices is found in a bio-cell which depends its reaction specificity under mild conditions on enzymes or microbial cells, and its energy conversion on electrochemical reactions on a pair of electrodes. The bio-cell should be the first bioelectronic device, although the bio-components are solubilized or suspended in solution.

In late 1960's, enzyme immobilization technology was emerged and led to develop an enzyme electrode. Enzyme is immobilized in a gel layer and attached to the platinum electrode surface to form an enzyme electrode. The first biosensor was demonstrated with the enzyme electrode comprising of glucose oxidase that oxidizes specifically glucose. Because the bioelectronic coupling is attained in a similar manner with the bio-cells, the biosensor rely its selectivity on enzyme and its signal transduction on electrochemical reaction. Other biocomponents including antibodies and cells have been implemented in a similar designing to develop such biodevices as immunosensors and microbial sensors.

It is noted that the bio-interfacing technology has been flourished in 1980's along with the uproading of the design concept of molecular devices. Although it has been faded away, the proposed idea of "biochip" gained too much attention and triggered intensive research and development of biodevices. Protein molecules were primary concerns with designing biodevices.

The expression of the protein functionality depends vitally on its conformation, i.e., the specific threedementional folding of the polymer chain. Most of the protein functionalities are attributed to a specific site of the surface domains. The functional parts that execute molecular recognition, specific catalytic reaction, and electron transfer are distinctly localized at a limited domain. This is a unique feature of proteins that is absent in smaller molecules. Enzymes, antibodies, and bacteriorhodopsin have successfully been incorporated, either as electronic or photonic components, to form biosensors or biophotonic devices. The molecular recognition function of these proteins has, however, been coupled with the electronic function of metal and semiconductors in an indirect manner because of the technical difficulty in electronically linking the electron transfer site of these proteins with the conventional electronic materials.

Even though a protein molecule can, in principle, work as a minimum electronic component, we have failed to utilize the electronic function of a single protein molecule. This failure is primarily due to a lack of fabrication technology for achieving a "biointerface". The biointerface establishes electronic communication at the molecular level between a protein molecule and a conventional electronic material, specifically, via electron transfer from one of the other. We have succeeded in establishing the electronic communication between protein molecules and a metal electrode by means of a novel design of the molecular interface. This accomplishment has opened a door for us to fabricate not only a new type of biosensor but also novel biodevices.

A marked progress in gene engineering and cellular engineering gave a distinctive impact on bioelectronics specifically in developing biodevices in 1990's. Gene engineering has made it possible to modify protein molecules 
as designed, and to produce unique protein derivatives that could fit to biodevices. The examples include many derivatives of bacterio rhodopsin, which are implemented in photonic biodevices, kimeric fusion proteins and lipidtagged antibody. Along with proteins, DNA has been implemented in biodevices, which should be characterized by "DNA chip". DNA-based biodevices, especially highly integrated microarray devices, have been rapidly commercialized and prevailed in the wider area of applications, of which primary concerns is genome analyses.

Both gene engineering and cellular engineering have provided us with a deep insight of the intercellular and intracellular biological information networks.

A living cell contains a whole set of intracellular information networks including gene information networks and signal transduction pathways. Molecular messengers such as hormones, cytokines and neurotransmitters are recognized by receptors embedded on the cell membrane surface, resulting in activation of the intracellular information networks mostly to modulate the gene information networks. On the basis of the biological information networks, living cells have successfully been implemented as a unit of biological information networks in biodevices. The cellular biodevices should be pursued in 2010's.

Another technical challenge confronting us is the electronic communication between living cells and a metal electrode. This difficulty has been partially overcome by our recent findings that the gene expression can be modulated by electrical stimulation when the cells are cultured on the electrode surface. We thus possess the basic technology to electronically link living cells to conventional electronic materials through an appropriate molecular interface. Such emerging technology has led us to design cellular biodevices.

In 2000's, a next step of bioelectronics evolution should be triggered by nanotechnology. One of the primary problems in development of nano-scale biodevices is how to access a single molecule, which should be solved by nanotechnology. Nanobiology is expected to offer novel candidates of biomolecular systems for biodevices.

Bioelectronics has been evolved every decade as

Table 1 Bioelectronic evolution by innovative technology in every decade.

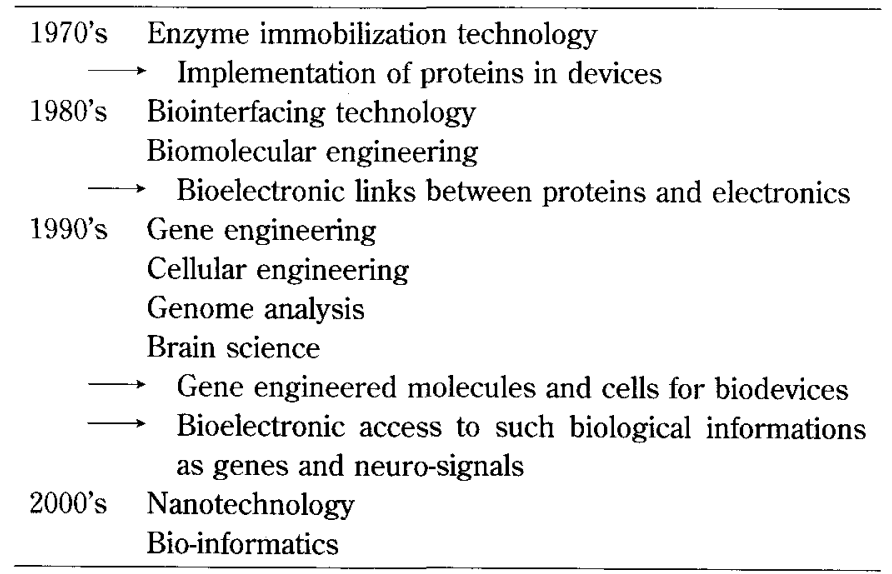

shown in Table 1, which is motivated by innovated technologies.

In this article, we shall first deal with the concept and design of the molecular interface for proteins, specifically redox enzymes, and their applications in fabricating biodevices. Second, we shall address the problem of electronic modulation of living cells, with the aim of designing an artificial neuron network for the construction of neurodevices.

\section{Biointerfacing Technology for Molecular Biodevices}

\section{1 Electronic and photonic molecular biodevices}

Protein-based molecular biodevices exploit either electronic or photonic properties of proteins. Electronic biodevices have been designed and constructed by utilizing such electroactive proteins as redox enzymes. One example is provided by a protein molecular rectifier comprised of two molecular layers of different proteins, which possess different electronic energy levels at their electron transfer sites. The protein molecular layer is electronically linked to the electrode surface. The electron flow between a pair of electrodes is rectified by the presence of the protein molecular layers.

Other electronic biodevices are biosensors comprised of redox enzymes with a selective recognition site for a specific determinant. The electron transfer following molecular recognition is effectively coupled between the redox site of the enzyme molecule and the electrode surface. The sensing function is based on the dependence of the electron transfer rate upon the concentration of a specific determinant.

Bacteriorhodopsin, which contains a retinal (vitamin A aldehyde) as its chromophore, bound to the protein within a restricted space, is a versatile protein that has been utilized for the construction of both photonic and electronic devices.

\section{2 Molecular interfacing technology}

Protein-based electronic devices are constructed in such a manner that an electroactive protein molecule is electronically linked with a metal electrode to establish an electronic communication. A biosensor comprised of a redox enzyme may be fabricated by immobilizing a layer of enzyme molecules on a working electrode, together with a counter electrode and, if necessary, a reference electrode in the vicinity. One surface of the enzyme layer faces the solution containing a determinant, and the other surface is attached to the electrode surface. The determinant is bound and recognized by the enzyme. This event is followed by electron transfer to or from the redox center of the enzyme. The electron transfer should be monitored by the electrode through a molecular interface.

Unfortunately, a very limited number of redox enzymes are capable of undergoing reversible electron transfer on the electrode surface. The electron transfer between redox enzymes and the electrode surface is restricted primarily because of the steric hindrance for electron access.

The advances in redox-enzyme-based electrodes have 
led us to conclude that a molecular level assembly should be designed to facilitate electron transfer at the interface between an enzyme molecule and a metal electrode. The molecular level assembly at the interface thus constitutes a molecular interface.

There are several molecular interfaces for redox enzymes.

1) Electron mediator: Either electrode or enzyme can be modified with an electron mediator. ${ }^{6,7)}$

2) Molecular wire: The redox center of an enzyme molecule can be connected to an electrode by means of a conducting polymer chain which form the molecular wire linking the redox center to the metal electrode. ${ }^{8,9)}$

3) Organic salt electrode and conducting polymer electrode: The surface of an organic electrode can provide enzymes with smooth electron transfer.

\section{3 Conducting polymer molecular wire for mo- lecular interface}

Conducting polymer molecular wires can be used as the molecular interface for redox enzymes on the electrode surface. Some of these conducting polymers can be electrochemically synthesized in aqueous solutions and deposited on the electrode surface. The thickness of the conducting polymer layer, i.e., the length of the molecular wire, can be controlled by adjusting the total electric charge passing through during electrolytic polymerization. Polypyrrole is one of the conducting polymers that is suitable for use as the molecular wire for redox proteins by means of the polaron mechanism. Glucose oxidase is one of the few redox enzymes that can be molecularly interfaced with a metal electrode by means of the molecular wire provided by polypyrrole. The molecular-interfaced glucose oxidase was prepared by the following procedure. ${ }^{8)}$

Glucose oxidase was first adsorbed onto the platinum surface at a regulated electrical potential. The protein adsorption varied, depending on several factors such as electrode potential, glucose oxidase concentration, $\mathrm{pH}$, and temperature. The effects of these factors on protein adsorption were carefully investigated.

Glucose oxidase gradually adsorbed onto the platinum electrode at a potential of $0.5 \mathrm{~V}$ vs. $\mathrm{Ag} / \mathrm{AgCl}$ (i.e., using the silver/silver chloride electrode as the reference) and pH 5.5. It reached a saturated state within $60 \mathrm{~min}$. Adsorbed glucose oxidase was assayed for its enzyme activity and its protein content. The results indicate that a monolayer of adsorbed glucose oxidase at a different surface coverage may be prepared under a controlled electrode potential. According to this characterization, a glucose oxidase monolayer was prepared at a surface coverage of $60-70 \%$ on the platinum electrode surface.

After rinsing, the adsorbed glucose oxidase was brought in contact with a solution containing pyrrole. The electrode potential was immediately set at $0.7 \mathrm{~V}$ vs. $\mathrm{Ag} / \mathrm{AgCl}$ to initiate pyrrole polymerization. Total charges passing through during polymerization were regulated with a coulombstat. Polypyrrole was deposited on the electrode surface in such a manner that the glucose oxidase intermolecular space could presumably be filled. Polymerization was terminated at an estimated membrane thickness of $2 \mathrm{~nm}$. With the assumption that glucose oxidase is a globular protein with a diameter of $3.5 \mathrm{~nm}$, the monolayer of glucose/polypyrrole on the electrode surface. Polypyrrole so strongly adheres to the electrode surface that the detachment of glucose oxidase from the platinum surface is prevented.

Other redox enzymes, including fructose dehydrogenase $(\mathrm{FDH})$ and alcohol dehydrogenase $(\mathrm{ADH})$, have been molecularly interfaced with a metal electrode by means of the polypyrrole conducting polymer according to a processes similar to what was described above. This process was found applicable to any size and shape of electrodes on which a monolayer of redox enzymes could be immobilized.

\section{4 Electron transfer of molecular-interfaced en- zymes}

Historically, electron transfer involving glucose oxidase (GOD) was difficult when it was adsorbed to the surface of a platinum electrode, although several papers suggest that electron transfer on the electrode surface is possible under uncertain conditions. In the above example, the molecular interface established by a conducting polymer presumably allow the active site of GOD, where flavin adenine dinucleotide (FAD) is bound as a prosthetic group, to be accessed electronically. This access allows electrons to be transferred between the active site and the electrode surface, to which the other end of the conducting polymer is attached. Glucose molecules diffuse into the active site of GOD, where GOD is oxidized to form gluconolactone. Concurrently, the GOD-bound FAD is reduced. In the case where no molecular oxygen is dissolved in the solution, the reduced form of GODbound FAD cannot transfer the electron elsewhere, and the oxidized form of GOD-bound FAD cannot be regenerated. However, an electron or a polaron may be transferred from the reduced form of GOD-bound FAD to the electrode via the molecular interface of a conducting polymer, if an appropriate gradient could be applied between the reduced form of GOD-bound $\mathrm{FAD}$ and the electrode.

Electron transfer of the molecularly interfaced glucose oxidase on the electrode surface was confirmed by differential pulse voltammetry and cyclic voltammetry. In the absence of dissolved molecular oxygen, the glucose oxidase clearly exhibited, in these voltammograms, both reductive and oxidative current peaks. These results indicate that electron transfer takes place from the electrode to the oxidized form of glucose oxidase and, subsequently, the reduced form is reoxidized by electron transfer to the electrode through polypyrrole. We therefore conclude that polypyrrole indeed works as a molecular wire between the adsorbed glucose oxidase and the platinum electrode.

\section{5 Electrically modulated activity of molecular-} interfaced enzymes

An enzyme possess two characteristic functions : molecular recognition and biocatalytic selectivity. The molecular recognition function is critically important for the design and construction of biomolecular devices, especially molecular sensing devices, because their selectivity 
depends on the molecular recognition of the enzyme. The biocatalytic function also has potential that can be exploited for designing novel devices. In the molecular sensing devices, the molecular information to be processed is transduced and amplified into an output electrical signal. However, this line of research is still quite primitive, and further investigation on implementation of the biocatalytic function is urgently needed.

Investigation of the molecular-interface of redox enzymes has yielded the following important findings. The molecular-interfaced redox enzymes exhibit dependence of the enzyme activity on the electrode potential. The enzyme is inactive when the electrode potential is set below a certain threshold. Once the electrode potential reaches the threshold, the enzyme activity increases with an increase in the electrode potential. The activity of the molecular-interfaced enzyme can be reversibly modulated by changing the electrode potential.

The molecular-interfaced glucose oxidase (GOD) catalyzes the cycling reaction. The substrate molecule, glucose, transfers an electron to the active site of GOD and is oxidized to gluconolactone. Since the prosthetic group FAD is located at the active site of GOD, it is reduced to $\mathrm{FADH}_{2}$. It is necessary to regenerate the oxidized form of the prosthetic group FAD so as to enable continuing catalytic reaction. If the electrode potential is lower, which means less negative, than the redox potential of the prosthetic group $\mathrm{FAD}$, then an electron will be transferred from the reduced form of $\mathrm{FAD}$ to the electrode via the molecular interface. The catalytic process can be cycled. Unless the energy balance is satisfied, the catalytic process will be inhibited.

The glucose oxidase/polypyrrole membrane electrode was immersed in a pH 5.5 citrate buffer solution containing potassium chloride, along with a counter electrode and an $\mathrm{Ag} / \mathrm{AgCl}$ reference electrode. The solution was bubbled with $\mathrm{N}_{2}$ gas so as to be deoxygenated prior to the experiment. The electrode potential was set to $0.0 \mathrm{~V}$ vs. $\mathrm{Ag} / \mathrm{AgCl}$, which is sufficient to oxidize the reduced form of $\mathrm{FAD}$ to the oxidized form.

After a steady anodic current was measured, glucose was added to the solution. The anodic current immediately increased as a result of oxidation of the reduced form of FAD, which is concurrent to the enzymatic oxidation of glucose in solution. These results show that the molecular function of glucose oxidase is transduced, with the aid of polypyrrole, to an electron flow to the electrode.

The anodic current increase is caused by the electrochemical oxidation of the enzymatically generated reduced form of glucose oxidase, and is therefore a measure of the enzyme activity. The enzyme activity of the molecular-interfaced glucose oxidase can be altered by the applied electrode potential. The enzyme activity instantly changes when the electrode potential is varied in the range from $0 \mathrm{~V}$ to $0.2 \mathrm{~V}$ vs. $\mathrm{Ag} / \mathrm{AgCl}$. Such a change is completely reversible. These results allow us to conclude that the enzyme activity of molecular-interfaced glucose oxidase can be controlled by the electrode potential.
The voltage dependence of the biocatalytic activity of the molecular-interfaced FDH is shown in a manner similar to the case of the molecular-interfaced glucose oxidase. ${ }^{10,11)}$ The output current corresponds to the enzyme activity of the molecular-interfaced FDH. The FDH remains inactive in the potential range below the redox potential of its prosthetic group PQQ. When the electrode potential exceeds the redox potential of the prosthetic group PQQ, the enzyme activity increases sharply with the increasing electrode potential.

The potential-controlled modulation of the molecularinterfaced enzymes may be interpreted as follows. The enzyme and its substrate molecule have their intrinsic redox potentials. The redox potentials of oxidases and dehydrogenases are determined by the prosthetic group, i.e., a cofactor such as $\mathrm{FAD}$, which resides at the active site of the enzyme. Due to a potential gradient, an electron can be transferred from the substrate molecule to the active site of the enzyme if the substrate molecule can fit into the molecular space around the enzyme's active site. However, the electron transfer between the active site of the enzyme and the electrode is regulated by the electrode potential, even if the molecular wire has been connected. It appears reasonable that the enzyme activity can be electrically modulated at a threshold of the redox potential of the prosthetic group of the enzyme.

\section{6 Molecular-interfaced proteins for electronic molecular biodevices}

The molecular interfacing technology has allowed such electroactive proteins as redox enzymes to electronically communicate with conventional electronic materials, including metal and semiconductors. The electronic communication has made it possible to transduce the molecular recognition of an enzyme substrate into an electronic signal, to switch on and off the enzyme activity, and to electrically modulate the enzyme activity. ${ }^{12)}$

On the basis of the molecular interfacing technology, the following biodevices have been developed.

(1) Electron-transfer-type biosensor

Either oxidase or dehydrogenase can be utilized for molecular recognition of a specific analyte in a sensing device. The molecular interface enables the sensing device to generate an electron flow between the enzyme and the analyte in response to molecular recognition. This type of biosensor is characterized by direct conversion of molecular recognition to electron transfer. Thus, the device implements counting of specific molecules by quantifying electrons.

(2) Molecular switch

The molecular-interfaced enzyme can be activated and inactivated by controlling the electrode potential. The enzyme therefore functions as a molecular switch in which an electric signal is transduced into a molecular signal.

(3) Biodevices for an intelligent bioreactor

The initial concept of a bioreactor is a reactor that works under mild conditions and without any side reactions, just like a living cell. The closest implementation of the bioreactor concept is accomplished by immobilizing a biocatalyst, although an autoregulative system has not 
Table 2 Gene engineered proteins for biodevices.

\begin{tabular}{ll}
\hline Probe-labeled protein & Luciferase/Protein A \\
& Lucirerase/RNA binding \\
& protein \\
\hline Lipid-tagged protein & Lipid/Single chain antibody \\
& Lipid/Protein A \\
\hline
\end{tabular}

been incorporated. The molecular-interfaced biodevices that contain biocatalysts allows for electrical regulation of biocatalytic activity in response to the changes of environmental conditions.

\section{Gene Engineering for Molecular Biodevices}

\section{1 Gene engineered proteins for biodevices}

Gene engineering may fall in three categories of engineering including gene designing of proteins, designing of gene expression process and gene analysis. Since gene engineering has made a marked progress, it has been successfully applied to develop biosensing material, which includes an enzyme-labeled binding protein for immunosensing and a lipid-tagged single chain antibody for immunosensing. ${ }^{13)}$ Further development has been under investigation.

\section{2 Protein A-luciferase fusion protein for lumi-} nescent biodevices

The protein A expression vector, pMPRA1, was previously constructed in our laboratory. The plasmid has a structural gene of Fc-binding domain of Staphylococcal protein A (from pRIT5) under the control of tac promoter, with possessing a multicloning site after the protein A gene, the $r m \mathrm{BT}_{1} \mathrm{~T}_{2}$ terminator, and $\beta$-lactamase gene. The plasmid, pT3/T7-1 luc, was digested with Bam HI to separate a $1.78 \mathrm{kbp}$ fragment containing a structural gene of firefly luciferase. The fragment was further digested with NarI, N-terminal 12 amino acids of corresponding protein were considered to be deleted. Despite the deletion of these amino acids, the expressed protein retained sufficient luciferase activity. The fragment encoding the luciferase gene was ligated into the multicloning site of pMPRA1. The resulting plasmid, protein A-luciferase fusion gene expression vector, was designated as pMALU2. ${ }^{(4)}$

The resulting fusion protein was designed by forming a site-to-site binding in a ratio of $1: 1$, i.e., the C-terminal site of protein A was fused to the N-terminal site of the deleted luciferase.

The binding capability of the fusion protein of luciferase and protein A to IgG was confirmed by Western blotting analysis.

The luciferase activity of the fusion protein was also confirmed.

Bioluminescent immunoassay for human IgG was performed. A series of human IgG solutions were prepared, and each solution was incubated in a cuvette whose surface was previously coated by $\mathrm{F}(\mathrm{ab})_{2}$ fragment of antihuman IgG antibody. The protein A-luciferase fusion protein was then incubated in the cuvette to carry out a sandwich-format immunoassay. After removing unbound fusion protein, the luciferase bound on the cuvette sur- face was determined by adding the solution of substrates. Human IgG was determined in the range from $10^{-3}$ to $10^{-7} \mathrm{~g} / \mathrm{ml}$ by the bioluminescent immunoassay using the genetically fused protein.

The protein A-luciferase fusion protein is very useful for the determination of trace amount of antigen or antibody in a very simplified and rapid manner using a photon-counting device. In addition, the fusion protein can be used as a universal marker in sandwich-format immunoassay, since the protein A moiety binds to the major subclasses of immunoglubulin.

One further point should be emphasized. This was the first example of constructing a protein A-firefly luciferase conjugate by recombinant gene technology.

\section{3 RNA binding protein-luciferase fusion protein}

Reverse transcription-polymerase chain reaction (RTPCR) has successfully been applied and evaluated in highly sensitive analysis of RNA with sequence specificity. The method is receiving increasing attention in DNA /RNA analysis as an alternative to standard methods including Northern blot analysis, nuclease protection assay, and in situ hybridization using radioisotopic labeling probes. It is noted that this method is specifically powerful for RNA having a low copy number. Reversetranscription and amplification of a sample RNA using a specific primer are effectively coupled with hybridization analysis. One hybridization analysis is based on the binding of protein along with the labels. Avidin and antibody have been commonly used in conjunction with the corresponding partners. In addition, it is efficient to use enzymes to enhance sensitivity and specificity.

We have isolated RNA binding protein from HeLa cell nuclear extract that bind specifically to single-stranded d(TTAGGG) 4 deoxyribonucleotide oligomer and more tightly to $\mathrm{r}$ (UUAGGG) 4 oligoribonucleotides, $\mathrm{rH} 4$. The protein exhibited no binding activity to rECGF oligoribonucleotide which has an unrelated sequence having the same length as $\mathrm{rH} 4$. The cDNA for the $\mathrm{rH} 4$ binding protein has been cloned and sequenced. ${ }^{15,16)}$ The primary structure predicted by the cDNA sequence reveals that this protein possesses two tandemly arranged RNA binding domains (RBDs) at its amino-terminus, and that the carboxyl-terminal region is particularly rich in glycine which appears to contribute to RNA binding. We have constructed the full-length recombinant protein and deleted mutant including two tandemly arranged RBDs (designated as $\mathrm{D} 12 \mathrm{H}$ ) to investigate the molecular mechanism of the sequence discrimination. The results indicated that all of these proteins could retain the RNA binding activity to recognize the $\mathrm{rH} 4$ with sequence specificity.

By fusing the structural genes of RBDs of r(UUAGGG) 4 binding protein, D12H, and firefly luciferase, a novel bifunctional protein has been constructed for the bioluminescent detection of $\mathrm{rH} 4$. The protein could be used to detect the membraneimmobilized $\mathrm{rH} 4$ rapidly and specifically. Moreover, when D12H of this protein is replaced by another RNA binding protein, the resulted fusion protein will be effective on the specific detection of different sequence RNAs. 
tween living cells and electronic materials. The author has devoted long and extensive efforts to the problem of electrical modulation of cellular function and has succeeded in achieving electric control of initiation and termination of cell proliferation, electric activation of gene expression, and electrically guided outgrowth of neurites. ${ }^{20-25)}$ These processes are important steps toward realizing cellular biodevices.

One of the most exciting findings involves astroglial cells in the brain. These cells support neuronal cells by secreting nerve growth factor (NGF). Astroglial cells are cultured on the surface of an optically transparent electrode, which is fixed at the bottom of a culture dish. A counter electrode and an $\mathrm{Ag} / \mathrm{AgCl}$ reference electrode are also installed in the culture dish, which is filled with a culture medium. A potentiostat sets the electrode poteintial at a fixed value with respect to the reference electrode.

Astroglial cells that had been cultured for 7 days in serum-free Dulbecco's modified eagle medium (DMEM) were subjected to another $1 \mathrm{hr}$ culture along with the application by $10 \mathrm{~Hz}$ of sine wave electrical potential at varying voltages. After the electrical stimulation, the cells were cultured for another $24 \mathrm{hr}$, the levels of secreted and intracellular NGF were determined. Intracellular levels of NGF remained unchanged by electric stimulation. In contrast, NGF secretion was extremely enhanced, reaching the maximum at an upper potential of $+0.3 \mathrm{~V}$.

The levels of NGF, c-fos, and c-jun mRNA in the electrically stimulated astroglial cells were quantified by competitive reverse transcription polymerase chain reaction (RT-PCR) assay. The results showed clearly that the electrical sine wave stimulation induces the c-fos, c-jun and NGF gene expressions in astroglial cells, and that the electrically stimulated NGF expression and excretion is triggered by the PKC-AP-1 pathway.

\section{4 Cellular biodevices for tissue alternative}

In the further investigation, hsp (heat shock protein) 70 promoter was found activated by the low frequency of electric stimulation, which led us to design an electrically responsive cells by gene engineering. Such an electrically responsive promoter plays a key role on designing the cell. A gene of interest is linked with hsp 70 promoter to form a plasmid, and is inserted in a cell. The engineered cell becomes electrically responsive, which results in expression of the gene of interest on call by electric stimulation. The hsp 70 promoter is electrically activated at first, which is followed by expression of the gene of interest.

The electrically responsive cells are applicable in designing cellular biodevices for tissue alternatives and drug excretion on demand.

\section{Concluding Remarks}

Several protein-based molecular biodevices have been made possible by the molecular interfacing technology that enables proteins to electronically communicate with the conventional electronic materials, such as metals or semiconductors.

Nanotechnology leads to a single molecular biodevices, of which a DNA-based single molecular biodevice seems promising.

Intensive investigation should be concentrated on cellular biodevices that has an innovative application in biosensing, cell therapeutics, and drug discovery.

\section{References}

1) Molecular Electronics - Science and Technology (Ed. A. Avirum), Engineering Foundation, New York (1989).

2) Molecular Electronics - Science and Technology (Ed. A. Avirum), American Institute of Physics, New York (1991).

3) Molecular Electronics; Biosensors and Biocomputers (Ed. F. T. Hong), Plenum Press, New York (1989).

4) Molecular Bioelectronics (Ed. C. Nicolini), World Sci. Pub., Singapore (1996).

5) Nanofabrication and Biosystems-Integrating Materials Science, Engineering, and Biology, (Ed. H. C. Hoch, L. W. Jelinski, H. G. Craighead), Cambridge Univ. Press, New York (1996).

6) A. E. Cass, G. Davis, G. D. Francis, H. A. O. Hill, W. J. Aston, I. J. Higgins, E. V. Plotkin, L. D. L. Scott, and A. P. F. Turner, Anal. Chem., 56, 667 (1984).

7) Y. Degani and A. Heller, J. Phys. Chem., 91, 6 (1987).

8) S. Yabuki, H. Shinohara, Y. Ikariyama, and M. Aizawa, J. Electroanal. Chem., 277, 199 (1990).

9) N. C. Foulds and C. R. Lowe, Anal. Chem., 60, 2473 (1988).

10) G. F. Khan, H. Shinohara, Y. Ikariyama, and M. Aizawa, J. Electroanal. Chem., 315, 263 (1991).

11) G. F. Khan, E. Kobatake, H. Shinohara, Y. Ikariyama, and M. Aizawa, Anal. Chem., 64, 1254 (1992).

12) M. Aizawa, Anal. Chim. Acta, 250, 249 (1991).

13) M. Aizawa, Y. Yanagida, T. Haruyama, and E. Kobatake, Sens. and Actuators B, 52, 204 (1998).

14) E. Kobatake, T. Iwai, Y. Ikariyama, and M. Aizawa, Anal. Chem., 208, 308 (1993).

15) Y. Kajita, J. Nakayama, M. Aizawa, and F. Ishikawa, J. Biol. Chem., 270, 22167 (1995).

16) Y. Kajita, E. Kobatake, F. Ishikawa, and M. Aizawa, $J$. Biotechnol., 43, 63 (1995).

17) K. Yun, E. Kobatake, T. Haruyama, M. L. Laukkanen, K. Keinanen, and M. Aizawa, Anal. Chem., 70, 260 (1998).

18) E. Kobatake, H. Sasakura, T. Haruyama, M. L Laukkanen, K. Keinenen, and M. Aizawa, Anal. Chem., 69, 1295 (1997)

19) G. H. Seong, T. Niimi, Y. Yanagida, E. Kobatake, and M. Aizawa, Anal. Chem., 72, 1288 (2000).

20) M. Yaoita, M. Aizawa, and Y. Ikariyama, Expl. Cell Biol., 57, 43 (1989).

21) M. Yaoita, Y. Ikariyama, and M. Aizawa, J. Biotechnol., 14, $321(1990)$.

22) J. Kojima, H. Shinohara, Y. Ikariyama, M. Aizawa, K. Nagaike, and S. Morioka, J. Biotechnol., 18, 129 (1991).

23) J. Kojima, H. Shinohara, Y. Ikariyama, and M. Aizawa, Biotechnol. Bioeng., 39, 27 (1992).

24) S. Koyama, T. Haruyama, E. Kobatake, and M. Aizawa, Nature Biotechnol ., 15, 164 (1997).

25) M. Aizawa, S. Koyama, K. Kimura, T. Haruyama, Y. Yanagida, and E. Kobatake, Electrochemistry, 67, 118 (1999). 